\title{
Nanoscale
}

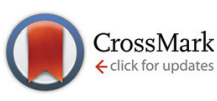

Cite this: Nanoscale, 2015, 7, 12860

\section{Quantum dot-layer-encapsulated and phenyl- functionalized silica spheres for highly luminous, colour rendering, and stable white light-emitting diodes $\uparrow$}

\author{
Hyein Yoo, ${ }^{a, c}$ Ho Seong Jang, ${ }^{b}$ Kwangyeol Lee ${ }^{c}$ and Kyoungja Woo*a,d
}

\begin{abstract}
Although the quantum efficiencies of quantum dots (QDs) are approaching unity through advances in the synthesis of QD materials, their luminescence efficiencies after mixing with resin and thermal curing for white light-emitting diodes (LEDs) are seriously lowered because of aggregation and oxidation of QDs and poor adhesion of QDs to the resin. To overcome these problems, QD-layer-encapsulated and phenyl-functionalized silica (SQS ${ }^{P h}$ ) spheres were synthesized and applied for white LEDs, whereby the QDs were homogeneously distributed at radial equidistance from the center and near the surface of approximately $100 \mathrm{~nm}$-sized silica spheres and the surface was functionalized with phenylethyl groups. The inter-core distances of QDs were over $\sim 14 \mathrm{~nm}$, which is over the limit $(<10 \mathrm{~nm}$ ) for Förster resonance energy transfer (FRET) that leads to photoluminescence (PL) reduction. This hierarchical nanostructure excludes a chance of FRET between QDs and provides the QDs a gradually refractive index matching environment, which yields $\sim 4$-fold enhanced PL in SQS ${ }^{\mathrm{Ph}}$. More importantly, the $S Q S^{\mathrm{Ph}}$ acquired a highly adhesive property to silicone resin due to their phenyl functional group matching, which resulted in remarkably improved light extraction in white LEDs. When incorporated along with a yellow-emitting $\mathrm{Y}_{3} \mathrm{Al}_{5} \mathrm{O}_{12}: \mathrm{Ce}^{3+}$ (YAG:Ce) phosphor and silicone resin on blue LED chips, the $\mathrm{SQS}^{\mathrm{Ph}}$ spheres presented significantly improved performance [luminous efficiency $(\mathrm{LE})=58.2 \mathrm{~lm} \mathrm{~W}^{-1}$; colour rendering index $\mathrm{Ra}=81.8 ; / / I_{0}=0.98$ after $60 \mathrm{~h}$ operation] than their original QDs $\left(\mathrm{LE}=39.6 \mathrm{Im} \mathrm{W}^{-1} ; \mathrm{Ra}=78.1 ; / / I_{0}=0.91\right.$ after $60 \mathrm{~h}$ operation) under a forward bias current of $60 \mathrm{~mA}$.
\end{abstract}

Received 7th May 2015 , Accepted 22nd June 2015 DOI: $10.1039 / \mathrm{c} 5 \mathrm{nr} 02991 \mathrm{k}$ www.rsc.org/nanoscale attracted attention for the protection of QDs from aggregation and oxidation. A QD-silica monolith substance, ${ }^{9}$ an irregularly shaped silica powder with embedded QDs, ${ }^{10}$ and a silicacoated composite film consisting of QDs and polymethylmethacrylate $^{11}$ have been packaged on blue LED chips. The QDs in the silica substance or powder were well dispersed, and the reported white LEDs including the silica-coated composite film exhibited highly improved luminescent stability compared with those without silica. However, the luminous efficiencies (LEs) of the resultant white LEDs were still much lower than the photoluminescence quantum yields (PL QYs) of the original QDs although they cannot be directly compared.

Meanwhile, QD-layer-encapsulated silica (SQS) sub-microspheres were reported with highly enhanced photoluminescence (PL, 2- to 3-fold) and stability based on their ligand-exchanged QDs. ${ }^{12}$ The QDs were homogeneously distributed without aggregation at radial equidistance from the centre and near the surface. By reducing the aggregation between SQS spheres, roughly $100 \mathrm{~nm}$-sized SQS spheres were improved to exhibit approximately 5 -fold enhanced PL based on their ligand-exchanged QDs and 4-fold enhanced PL based 
on their original QDs. ${ }^{13}$ However, the mechanism for PL enhancement is not yet known.

Nevertheless, the SQS spheres can be a good starting material to overcome the aggregation and oxidation problems of QDs for white LEDs because the QDs are homogeneously distributed and the silica shell provides some stability to QDs. ${ }^{12}$ More importantly, for a good adhesion to silicone resin, the surface functional group of SQS spheres can be facilely matched with the key functional group (i.e., a phenyl group) of a silicone resin, which is expected to improve the LE of the resultant white LEDs. Also, determining the mechanism for PL enhancement is important not only for fundamental science but also for reliable application in various fields.

Today, the most prevalent white LED in solid-state lighting is composed of a blue LED chip and yellow-emitting $\mathrm{Y}_{3} \mathrm{Al}_{5} \mathrm{O}_{12}$ : $\mathrm{Ce}^{3+}$ (YAG:Ce) phosphor and provides high LE with low cost. ${ }^{8,14,15}$ In this device, a part of the blue light emitted from InGaN chip is absorbed by YAG:Ce phosphor and converted to yellow light. Thus, the resultant white light consists of blue and yellow, showing a critical drawback: red-deficiency. There have been efforts to improve the colour rendering index (CRI) by incorporating red-emitting QDs as phosphors, which exhibited improved CRIs but seriously lowered LEs due to their intrinsic trade-off pairwise. ${ }^{8,15-18}$ Doped QDs with large Stokeshift (CdSe:Cu/ZnS with PL QY up to 50\%) were introduced as a promising phosphor, improving CRI with reduced sacrifice in LE because they exclude a chance of reabsorption originating from multiphase phosphors. ${ }^{19}$ However, commercial QDs with CdSe/ZnS core/multi-shell structure showing high PL QY $(\sim 70 \%)$ and stability are predominant and conveniently available. Thus, in the current case, it is deserved to apply commercial QDS for the preparation of red-emitting SQS. More importantly, by surface-engineering the resultant SQS with phenyl, and by incorporating it into a commercial red-deficient white LED, it may be possible to achieve much higher LE and stability than the case incorporating the original commercial QDs, along with a high CRI.

In this report, we present QD-layer-encapsulated and phenyl-functionalized silica $\left(\mathrm{SQS}^{\mathrm{Ph}}\right)$ along with a proposed mechanism for PL enhancement and its application for white LEDs yielding significantly improved performance $(\mathrm{LE}=$ $58.2 \mathrm{~lm} \mathrm{~W}^{-1}$; average CRI Ra $=81.8 ; I / I_{0}=0.98$ after $60 \mathrm{~h}$ operation) than the case incorporating the original commercial QDs $\left(\mathrm{LE}=39.6 \mathrm{~lm} \mathrm{~W} \mathrm{~W}^{-1} ; \mathrm{Ra}=78.1 ; I / I_{0}=0.91\right.$ after $60 \mathrm{~h}$ operation) under a forward bias current of $60 \mathrm{~mA}$.

\section{Experimental}

\section{Materials}

Octadecylamine-protected CdSe/ZnS core/multi-shell quantum dots $\mathrm{QD}_{1}$ and $\mathrm{QD}_{2}\left(\lambda_{\text {emit }}=611 \mathrm{~nm}\right.$ and $620 \mathrm{~nm}$, respectively; PL QY by vendor $\sim 70 \%$ for each; average diameter \pm standard deviation $=9.2 \pm 0.8 \mathrm{~nm}$ and $10.0 \pm 1.4 \mathrm{~nm}$, respectively over 100 particles) were purchased from Nanosquare Inc., Seoul, Korea. Silica spheres $(5.82 \%$ in water, average diameter \pm standard deviation $=50.7 \pm 4.2 \mathrm{~nm}$ over 100 particles, Polysciences, Inc.), $\mathrm{NH}_{4} \mathrm{OH}$ (28.0-30.0\%, Junsei Chemical Co.), $\mathrm{NaOH}$ (93\%, Showa Chemical Co., Ltd), tetraethylorthosilicate (TEOS, 98\%, Sigma Aldrich), 3-(aminopropyl)trimethoxysilane (APTMS, 97\%, Sigma Aldrich), and 3-mercaptopropionic acid (MPA, 99\%, Sigma Aldrich), trimethoxy(2-phenylethyl)silane (TMPES, 98\%, Sigma Aldrich) were used as purchased.

\section{Synthesis and characterization of SQS and SQS ${ }^{\mathrm{Ph}}$}

SQS particles were synthesized by following a previously described method. ${ }^{13}$ Briefly, the purchased silica particle solution $(20 \mathrm{~mL})$ was centrifuged after addition of $20 \mathrm{~mL}$ of ethanol and the solid was dispersed in $40 \mathrm{~mL}$ of methanol. After adding APTMS ( $~ 5 \%$ of silica), the solution was refluxed overnight for amine-functionalization. The product was washed with ethanol, dispersed in $23.2 \mathrm{~mL}$ of ethanol and used whenever needed (5\% stock solution). One $\mathrm{mL}$ of the stock solution was centrifuged and the solid was dispersed in deionized water (DW). The $\mathrm{pH}$ was adjusted to $\sim 4$ to yield $5 \mathrm{~mL}$ of solution $\mathrm{S}$. The carboxy-terminated QD solution was freshly prepared from purchased QDs $\left(1.0 \times 10^{-5} \mathrm{M}, 4 \mathrm{~mL}\right.$ in $\mathrm{CHCl}_{3}$ ) by adding $0.1 \mathrm{M} \mathrm{MPA}$ containing $0.12 \mathrm{M} \mathrm{NaOH}$ in methanol (1.6 mL) and stirring for $15 \mathrm{~h}$. To this solution, $4 \mathrm{~mL}$ of DW was added and the resultant QD-MPA in the upper layer was taken and centrifuged after addition of a $4: 1$ mixture of ethyl acetate and methanol. The solid was dispersed in DW and the solution was adjusted to $\mathrm{pH} \sim 10$ to yield solution $\mathrm{Q}$ $\left(1.0 \times 10^{-6} \mathrm{M}, 40 \mathrm{~mL}\right)$. Solution $\mathrm{S}(3.75 \mathrm{~mL})$ was added dropwise into $30 \mathrm{~mL}$ of solution $\mathrm{Q}$ with gentle shaking. The resultant SQ solution was centrifuged and the solid was dispersed in ethanol $(600 \mathrm{~mL})$ for encapsulation with silica. After adding DW $(3.6 \mathrm{~mL}), \mathrm{NH}_{4} \mathrm{OH}(2.4 \mathrm{~mL})$, and TEOS $(0.6 \mathrm{~mL})$, the solution was stirred for $3 \mathrm{~h}$. After the first $1 \mathrm{~h}$, the solution was sonicated every $30 \mathrm{~min}$ for $1 \mathrm{~min}$ to avoid aggregation between intermediate SQSs. The solid SQS was separated and washed with ethanol three times using centrifugation. Finally, the SQS solid was dispersed in $60 \mathrm{~mL}$ of ethanol ([QD] $\sim 5 \times 10^{-7} \mathrm{M}$ ). For phenyl-functionalization of SQS, the SQS solution $(20 \mathrm{~mL})$ was diluted in a mixture of ethanol $(114 \mathrm{~mL})$ and chloroform $(67 \mathrm{~mL}) . \mathrm{NH}_{4} \mathrm{OH}(0.134 \mathrm{~mL})$ and $\mathrm{DW}(0.2 \mathrm{~mL})$ were added with stirring. After $10 \mathrm{~min}$, TMPES $(0.02 \mathrm{~mL})$ was added and the solution was stirred for $15 \mathrm{~h}$. To minimize the aggregation during phenyl-functionalization, the solution was treated in a sonication bath for $1 \mathrm{~min}$ every $30 \mathrm{~min}$ after $5 \mathrm{~h}$. Finally, the SQS $^{\mathrm{Ph}}$ was washed with ethanol and chloroform using centrifugation and then dispersed in $20 \mathrm{~mL}$ of chloroform ([QD] $\sim 5 \times$ $10^{-7} \mathrm{M}$ ). To estimate the QD concentration in a consecutive synthetic process, we assumed $100 \%$ yield because the supernatants were almost colourless under UV; however, there must be at least a minimal experimental loss.

UV-Vis absorption and PL spectra were obtained using a Perkin Elmer Lambda 25 and a Hitachi F-7000 spectrophotometer with a QD-based concentration of $5 \times 10^{-8} \mathrm{M}$ in all the traces using ethanol or chloroform as a solvent. The PL data were recorded with $485 \mathrm{~nm}$ or $550 \mathrm{~nm}$ excitation. PL QY data were recorded using a Hamamatsu Photonics C9920-02 
absolute PL QY measurement system at Korea Institute of Machinery \& Materials for the original QD, SQS, and SQS ${ }^{\mathrm{Ph}}$ solutions only, because these solutions were stable for more than 1 year whereas $Q$ and SQ solutions were not stable even after 1 day. FT-IR spectra were obtained using a Mattson IR 300 spectrometer with a $\mathrm{KBr}$ pellet including each sample. TEM and SEM images were obtained using a Philips CM30 with $200 \mathrm{kV}$ and FEI Inspect F50 with $15 \mathrm{kV}$, respectively.

\section{Fabrication and characterization of SQS- or SQS $^{\mathrm{Ph}}$-incorporated white LEDS}

White LEDs were fabricated by combining InGaN-based blue LED chips $\left(\lambda_{\max }=440 \mathrm{~nm}\right.$, non-encapsulant-molded 5630 package mounted on Al PCB, LG Innotek, Korea) with the mixtures composed of as-dried QDs or SQS analogues, YAG:Ce phosphor (Intematix Co., NYAG4156) and silicone resin (Dow Corning Co., OE-6630 A/B KIT). The relative weight ratio in the mixture was adjusted to obtain white light as 100:10:0.7 (single product) or 0.75 (mixture) for resin :YAG:Ce : total SQS analogues, and as 1:2 for $\mathrm{SQ}_{1} \mathrm{~S}: \mathrm{SQ}_{2} \mathrm{~S}$ and $\mathrm{SQ}_{1} \mathrm{~S}^{\mathrm{Ph}}: \mathrm{SQ}_{2} \mathrm{~S}^{\mathrm{Ph}}$. When the original QDs were incorporated, the relative ratio was adjusted to $100: 10: 0.01$ for resin:YAG:Ce:QD. The slowly agitated mixture was transferred into the reflector of the 5630 package, and the combined package was treated in an oven at $60{ }^{\circ} \mathrm{C}$ for $1 \mathrm{~h}$ and then at $150{ }^{\circ} \mathrm{C}$ for $1 \mathrm{~h}$. Later, it was confirmed that the white LEDs treated at $150{ }^{\circ} \mathrm{C}$ for $2 \mathrm{~h}$ showed the same performance. Optical properties such as the LE, CRI, correlated colour temperature (CCT), and the CIE colour coordinates of the fabricated white LEDs were evaluated using a WITHLIGHT OPI-100 system with an integrating sphere under a forward bias current of $60 \mathrm{~mA}$ at room temperature.

\section{Results and discussion}

For a red-emitting $\mathrm{SQS}^{\mathrm{Ph}}$ with highly enhanced PL, roughly $100 \mathrm{~nm}$-sized SQS particles with emission peaks at $611 \mathrm{~nm}$ and $620 \mathrm{~nm}$ were prepared following a previously described method, ${ }^{13}$ and their surface was functionalized via a sol-gel reaction of trimethoxy(2-phenylethyl)silane (TMPES) according to Scheme 1. Briefly, ligand-exchanged QDs with mercaptopropionic acid (Qs) were self-assembled on an aminopropyl-functionalized silica sphere (S) using electrostatic interaction, yielding an SQ solution. The SQ solution was centrifuged and the solid SQ was dispersed in ethanol. A subsequent sol-gel reaction using tetraethoxysilane (TEOS) produced a silica shell on the SQ, yielding an SQS solution, and the final sol-gel reaction using TMPES on SQS yielded a phenylethyl-functionalized SQS. In this report, phenylethyl- or phenyl-functionalized SQS will be used and denoted as $\mathrm{SQS}^{\mathrm{Ph}}$ because its key function comes from the phenyl moiety.

A representative set of data for $\mathrm{SQS}^{\mathrm{Ph}}$ emitting at $620 \mathrm{~nm}$ is displayed in Fig. 1 and S1 (ESI†), and a set of data for SQS ${ }^{\mathrm{Ph}}$ emitting at $611 \mathrm{~nm}$ is included in Fig. S2. $\dagger$ The clockwise TEM images in Fig. 1a distinctly show each material corresponding to Scheme 1. That is, the darker QD particles are homogeneously distributed on a grey silica particle in SQ and the SQ was encapsulated with a thin silica shell around $10-15 \mathrm{~nm}$ to yield an SQS. In the SQS and SQS ${ }^{\mathrm{Ph}}$, the encapsulated QDS appear in the form of a dark ring and scattered dots within the ring because the TEM data exhibit projected images of the three-dimensional SQS and $\mathrm{SQS}^{\mathrm{Ph}}$ on a two-dimensional plane. Thus, the ring pattern can be an indicator for homogeneous distribution of QDs as a layer within a large silica sphere. Within the ring pattern, the dark grey dots may reflect the QDs from the lower hemisphere and the distinctly darker dots may reflect the QDs from the upper hemisphere.
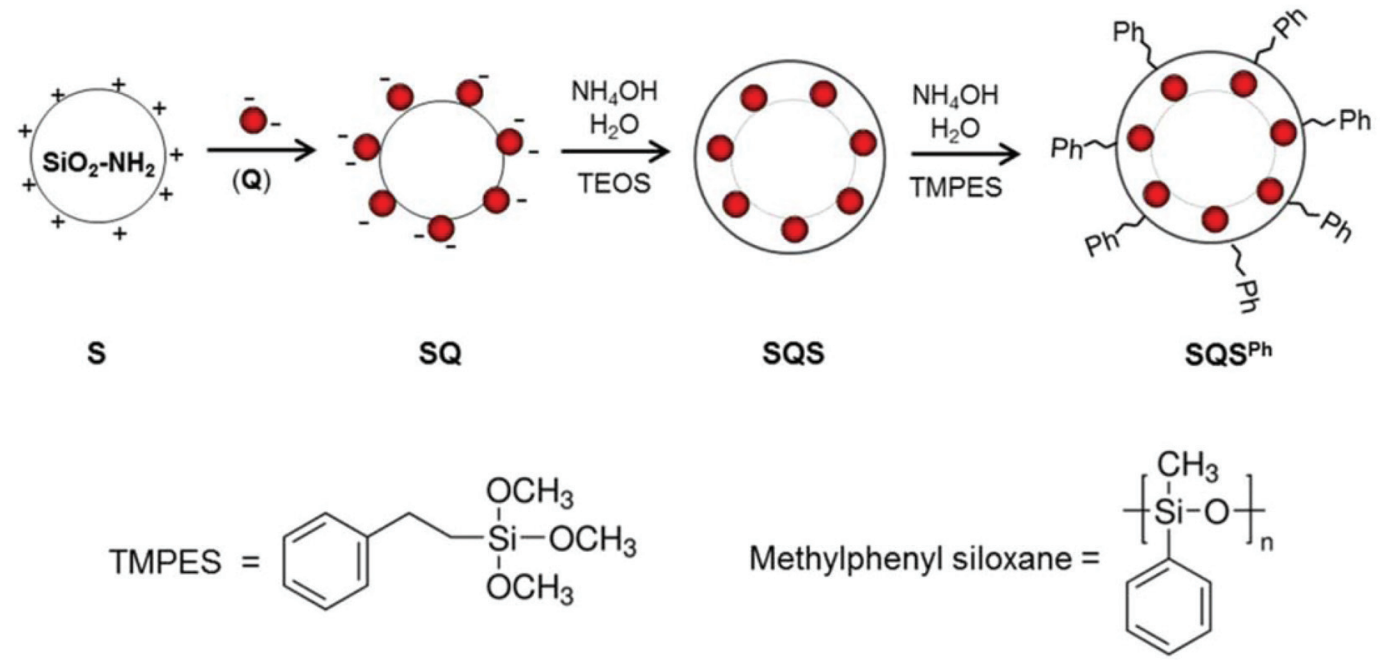

Scheme 1 Synthesis of QD-layer-encapsulated and phenyl-functionalized silica (SQS ${ }^{\mathrm{Ph}}$ ) spheres and chemical structure of a hardening component (i.e., methylphenyl siloxane) of a silicone resin. 
(a)

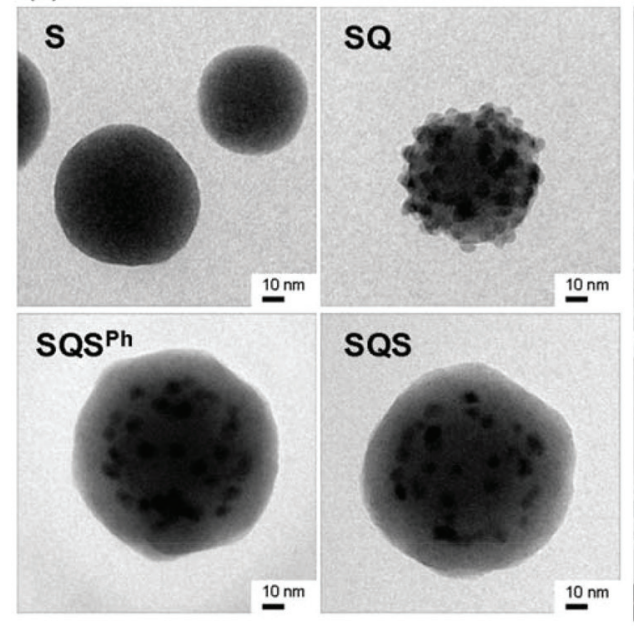

(b)

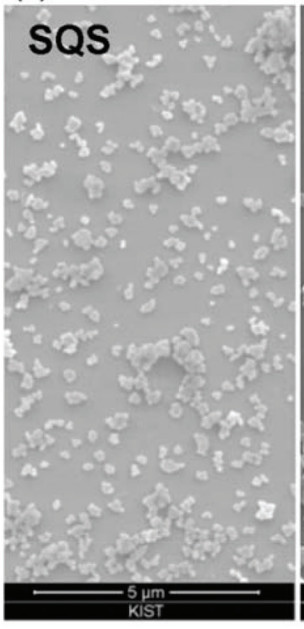

(c)

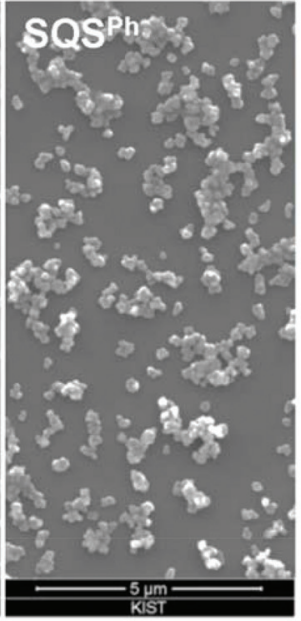

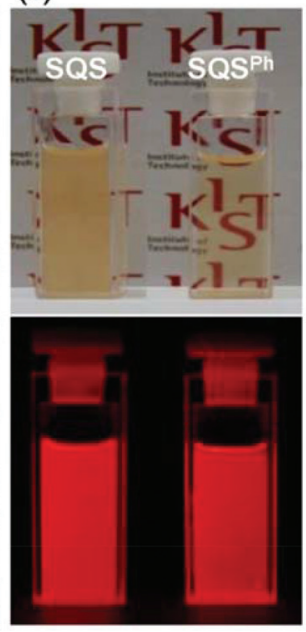

(d)

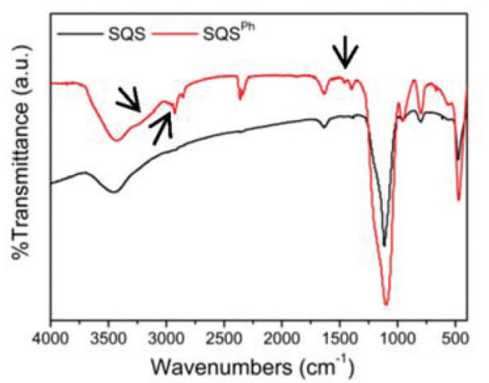

(e)

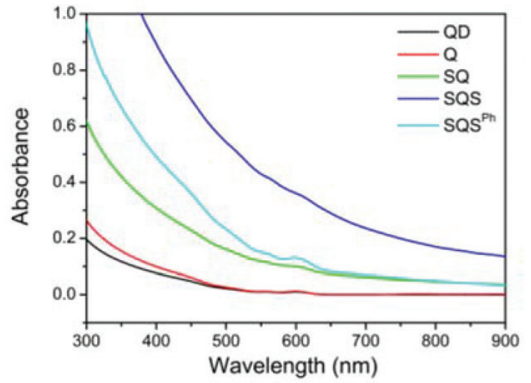

(f)

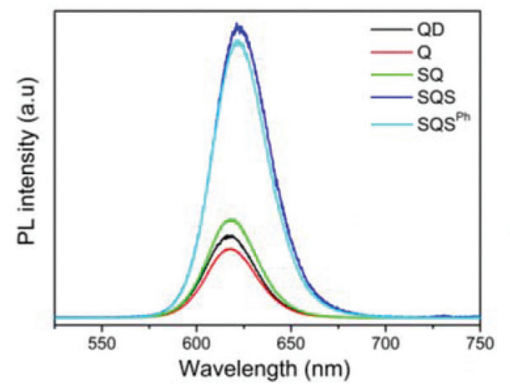

Fig. 1 (a) TEM images, (b) SEM images, and (c) photographs, and (d) FT-IR, (e) UV-Vis absorption, and (f) PL spectra of the materials (QD, Q, S, SQ, SQS, and SQS ${ }^{\mathrm{Ph}}$ with $\lambda_{\mathrm{emit}}=620 \mathrm{~nm}$ ) corresponding to Scheme 1 .

Additional images to show a homogeneous distribution can be found in Fig. S2 $\uparrow$ and a previous report. ${ }^{12}$ The TEM image of $\mathrm{SQS}^{\mathrm{Ph}}$ is not distinguishable from that of SQS because the organic monolayer consisting of phenylethyl is too thin to be observed. As can be noticed in the SEM images, intermittent sonication of the solution during silica shell formation greatly reduced the aggregation of roughly $100 \mathrm{~nm}$-sized SQS particles (Fig. 1b, left) compared with the case without sonication (Fig. S1b†). Notably however, the $\mathrm{SQS}^{\mathrm{Ph}}$ particles exhibited slightly higher aggregating tendencies than the SQS particles (Fig. 1b). The aggregation of SQS ${ }^{\mathrm{Ph}}$ particles appears to be caused by the temporary stacking of peripheral phenyl groups between the neighbouring $\mathrm{SQS}^{\mathrm{Ph}}$ particles. The phenyl groupstacking is an indicator of good mixing and adhesion of $\mathrm{SQS}^{\mathrm{Ph}}$ to the silicone resin, as will be discussed later. In the photographs of Fig. 1c, the SQS particles produce an opaque solution in ethanol, whereas the $\mathrm{SQS}^{\mathrm{Ph}}$ particles produce a transparent solution in chloroform because of their higher solubility after phenyl-functionalization. A better solvent was not found for the SQS particles. Nevertheless, both solutions exhibited highly bright red emissions.

The new FT-IR peaks appearing at approximately $3100 \mathrm{~cm}^{-1}$ (shoulder), slightly below $3000 \mathrm{~cm}^{-1}$, and $1453 \mathrm{~cm}^{-1}$ in SQS in Fig. 1d represent the vibrational stretching modes of aromatic and aliphatic $\mathrm{C}-\mathrm{H}$ and the bending mode of $\mathrm{CH}_{2},{ }^{20}$ respectively, and implicate surface-functionalization of SQS with the phenylethyl moiety. The absorption and PL spectra in Fig. 1e and $\mathrm{f}$ exhibit the same trends as those ${ }^{12,13}$ previously reported for SQS. Both the absorption and PL intensities of SQ and SQS increased moderately to dramatically compared with those of the original QDs in the order QD $<\mathrm{SQ}<\mathrm{SQS}$. Here, it is noteworthy that we assumed $100 \%$ yield to estimate the QD concentration in a consecutive synthetic process; however, there must be at least a minimal experimental loss that may contribute to decrease the absorption and PL intensities, which is in contrast to the current data. The increasing trend of the absorbance is partially attributable to the light scattering by aggregated and poorly soluble particles in the opaque solution. Thus, the PL intensity is expected to exhibit a further decreasing trend as the synthetic process proceeds. However, the SQS solutions encapsulating QDs with $\lambda_{\text {emit }}=620 \mathrm{~nm}$ and $611 \mathrm{~nm}$, respectively, exhibited 3.9- and 3.7-fold enhanced PL compared with their original QD solutions. After phenylfunctionalization, the absorbance spectrum of $\mathrm{SQS}^{\mathrm{Ph}}$ becomes much lower than that of SQS, whereas their PL intensities are comparable. Despite the temporary slight aggregation by 
phenyl group-stacking, the lowered absorbance spectrum and comparable PL intensity of SQS ${ }^{\mathrm{Ph}}$ compared with those of SQS are attributable to the high solubility of $\mathrm{SQS}^{\mathrm{Ph}}$ in chloroform. This behavior is clearly recognizable in the photographs of Fig. 1c.

The PL QYs of the original QD, SQS, and $\mathrm{SQS}^{\mathrm{Ph}}$ solutions were $69.0 \%, 47.4 \%$, and $34.7 \%$, respectively. As described earlier, because the SQS solution is opaque, its PL QY may not be reliable. Anyway, it is certain that the PL QY of $\mathrm{SQS}^{\mathrm{Ph}}$ is lower compared with that of the original QDs. However, the lower PL QY of the solution does not necessarily mean the lower LE of the resultant white LED as will be discussed later.

After careful study, we reasoned that the moderate PL enhancement (1.2-fold) achieved by forming SQ is attributable to the nanostructured rough surface of silica. It has been known that the light extraction efficiency from an emitter increases through surface roughening because the refractive index (RI) of a roughened surface appears as an intermediate between those of the surrounding medium (here, solvent) and the material without surface roughening and thus, the resultant gradual RI matching effect reduces internal light reflection and scatters the light outward. ${ }^{21-24}$ The local area of the silica sphere where a QD is located can be approximated as a roughened surface because the amorphous silica prepared by the Stöber process ${ }^{25}$ consists of a porous network structure with a porosity of $10-15 \%{ }^{26-28}$ In an on-going study, we confirmed that the SQ analogue consisted of a further roughened silica sphere and the original QDs showed 2.7-fold enhanced PL based on the original QDs. It supports our reasoning for PL-enhancement by surface roughening and the result will be published elsewhere after further study. The additional PL enhancement from SQ to SQS is also attributable to the gradual RI matching effect by the silica shell whose RI is intermediate between those of QD and the surrounding medium (solvent); however, the enhancement (3.9-fold) is superior to the case of SQ (1.2-fold) because the thin (10-15 nm) silica shell covers most part of the QD surface.

Furthermore, by virtue of structural characteristics, the SQS appears to exhibit the gradual RI matching effect efficiently. In the SQS, the QDs are homogeneously distributed due to electrostatic interaction at radial equidistance near the surface of the silica sphere. The average inter-QD-distance was over $20 \mathrm{~nm}$ in a previous report ${ }^{12}$ and is over $10 \mathrm{~nm}$ here because much larger QDs are used (i.e., $10.0 \mathrm{~nm}$-sized QDs are used here compared with the previous case where $6.0 \mathrm{~nm}$-sized QDs were used). Accordingly, considering the relationship between the diameter and band edge absorption wavelength of CdSe cores, ${ }^{29}$ the average inter-core-distance of QDs is estimated over $14 \mathrm{~nm}$. This distance is over the limit for the Förster resonance energy transfer (FRET $<10 \mathrm{~nm}$ ) which leads to serious reduction in PL intensity by self-quenching between QDs. ${ }^{3,10,28,30,31}$ It is in contrast with other studies that reported PL reduction by FRET in QD-embedded silica. ${ }^{9,10,32}$ Also, the homogeneous distribution of QDs at radial equidistance of the silica sphere is efficient for the light extraction compared with the accumulated one along the radial direction. ${ }^{12,32}$ All these structural characteristics of the SQS play an important role in efficient extraction of PL by gradual RI matching.

It is also noteworthy that light absorption in SQ and SQS can be enhanced by gradual RI matching, too. ${ }^{24}$ The absorbance values of $\mathrm{QD}, \mathrm{Q}, \mathrm{SQ}, \mathrm{SQS}$, and $\mathrm{SQS}^{\mathrm{Ph}}$ at $485 \mathrm{~nm}$ in Fig. 1e were $0.027,0.033,0.177,0.582$, and 0.261 , respectively. In the cases of opaque SQ and SQS solutions, the increased absorbance values appeared as a summation of the enhanced light absorption by gradual RI matching and the light scattering caused by their aggregation and poor solubility, which were not separable. On the other hand, in the case of transparent $\mathrm{SQS}^{\mathrm{Ph}}$, the enhanced light absorption was reflected as the major increment in absorbance value by approximately 9.7fold based on the QDs, in Fig. 1e. (The absorbance value by silica only solution ${ }^{12}$ was ignorable compared with those by SQ, SQS, and SQS ${ }^{\mathrm{Ph}}$.) Thus, the lowered PL QY of $\mathrm{SQS}^{\mathrm{Ph}}$ solution $(34.7 \%)$ based on the original QDs $(69.0 \%)$ is attributable to the relatively high enhancement in light absorption than in PL. Nevertheless, the remarkably enhanced PL with the proposed mechanism makes the SQS analogues as a promising candidate for phosphors and additionally, for other applications such as bio-imaging and bio-sensing materials.

Before white LED packaging, we simply investigated the miscibility and adhesive property of SQS analogues to silicone resin by mixing and curing them as a monolithic plate. The backbone of the hardening component (i.e., methylphenyl siloxane) of a commercial silicone resin (OE-6630 A/B KIT) has many dangling phenyl groups as illustrated in Scheme 1. In Fig. 2a, the SQS contains some aggregated dots that are detectable by the naked eye, as indicated by the arrows. However, $\mathrm{SQS}^{\mathrm{Ph}}$ exhibits superior miscibility and adhesion to the resin than SQS because $\mathrm{SQS}^{\mathrm{Ph}}$ and resins have the same phenyl groups, and the phenyl groups are alternately stackable.

Fig. $2 \mathrm{~b}$ presents a representative image and diagram of the white LED package that was prepared by integrating SQS or $\mathrm{SQS}^{\mathrm{Ph}}$ along with YAG:Ce phosphor and silicone resin on a blue LED chip. For comparison, nine different white LED samples were fabricated using different materials as the luminescence down-converters on blue LED chips. Their optical properties were investigated using a forward bias current of $60 \mathrm{~mA}$, and the results are summarized in Table S1. $\dagger$ Here, the original QDs emitting at $611 \mathrm{~nm}$ and $620 \mathrm{~nm}$ are denoted as $\mathrm{QD}_{1}$ and $\mathrm{QD}_{2}$, respectively. The corresponding ligand-exchanged QDs and their derived products are denoted as $\mathrm{Q}_{1}$ and $\mathrm{Q}_{2}, \mathrm{SQ}_{1} \mathrm{~S}$ and $\mathrm{SQ}_{2} \mathrm{~S}$, and $\mathrm{SQ}_{1} \mathrm{~S}^{\mathrm{Ph}}$ and $\mathrm{SQ}_{2} \mathrm{~S}^{\mathrm{Ph}}$, respectively. When only the YAG:Ce phosphor was integrated for the white LED, the resultant CRI (Ra), which is the average value of R1-R8, and LE were 65.2 and $78.9 \mathrm{~lm} \mathrm{~W}^{-1}$, respectively. When the original QD was integrated along with the YAG:Ce phosphor, the respective Ra and LE were 78.1 and $39.6 \mathrm{~lm} \mathrm{~W}{ }^{-1}$ for $\mathrm{QD}_{1}$ and 70.6 and $37.1 \mathrm{~lm} \mathrm{~W}^{-1}$ for $\mathrm{QD}_{2}$. As expected from a reference, ${ }^{8}$ a moderate increase in Ra and sharp decrease in LE were observed most likely because of the aggregation of QDs. The white LEDs with original QDs can be used as standards to compare the optical properties of the fabricated LEDs with SQS analogues. When SQSS were incorpor- 
(a)

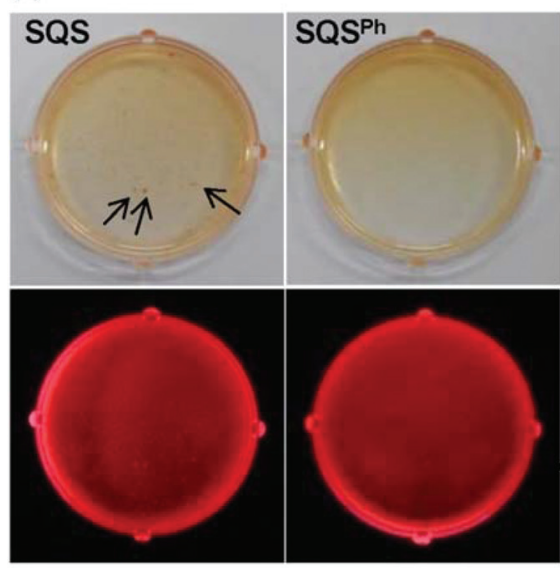

(c)

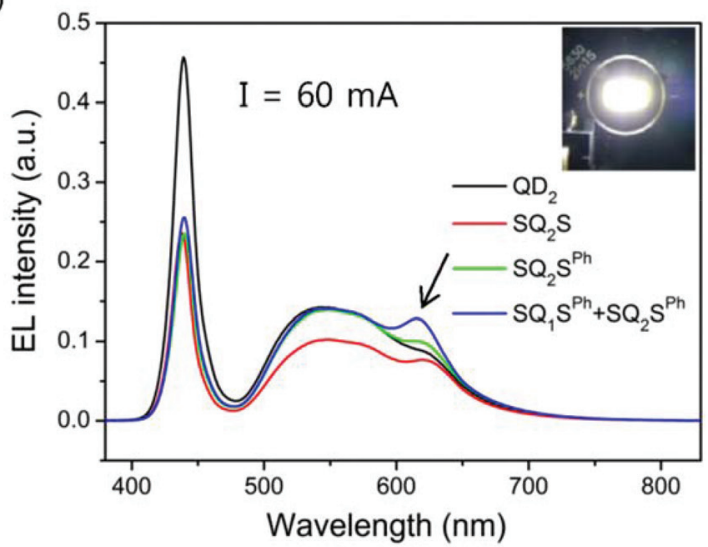

(b)

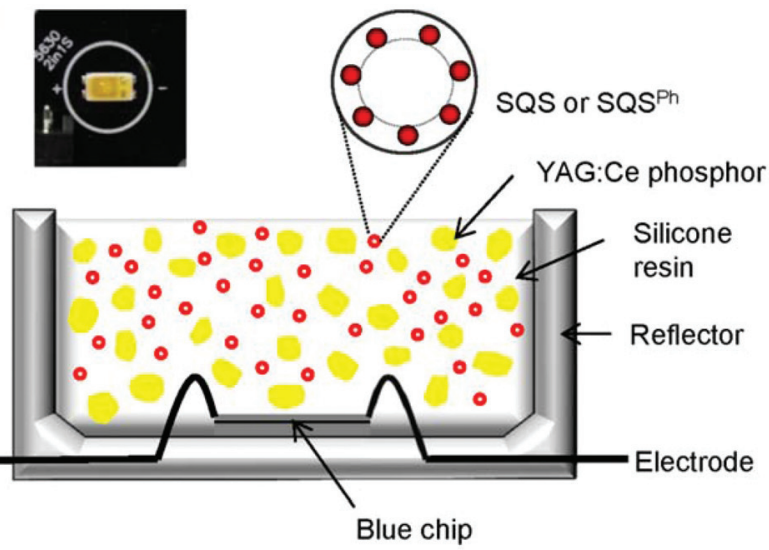

(d)

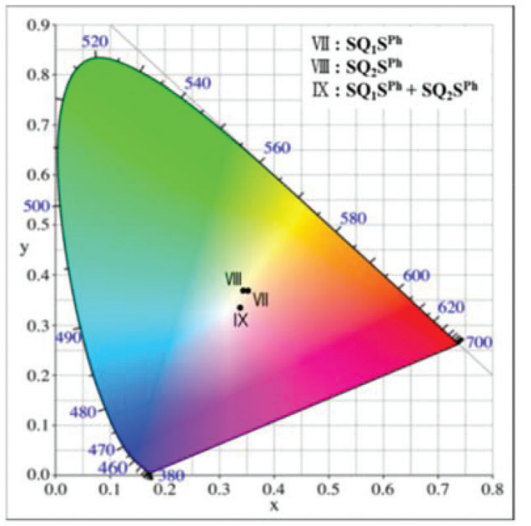

(f)

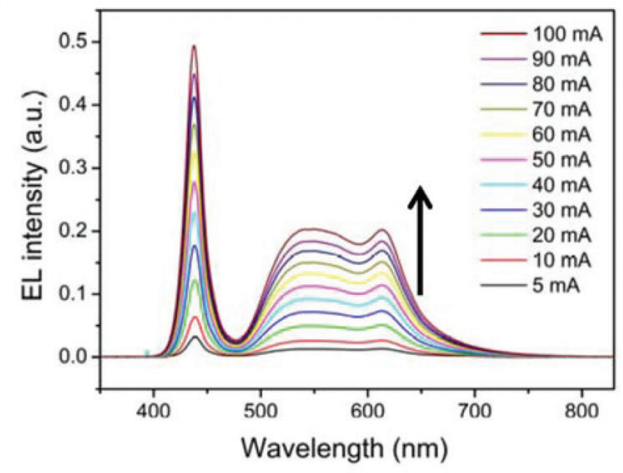

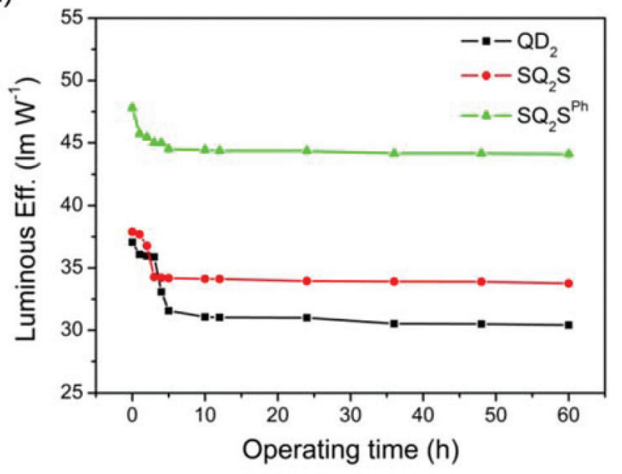

Fig. 2 (a) Photographs of the monolithic plates comprised of silicone resin and SQS or SQS ${ }^{\mathrm{Ph}}$, (b) photograph and diagram of an SQS ${ }^{\mathrm{Ph}}$ - and YAG: Ce phosphor-based white LED, (c) EL spectra of various down-convertor- and YAG:Ce phosphor-based white LED samples and an SQS ${ }^{\text {Ph }}$ mixtureand YAG:Ce phosphor-based white LED in operation (inset), (d) CIE color coordinates of various SQS ${ }^{\mathrm{Ph}}$ - and YAG:Ce phosphor-based white LEDs at $60 \mathrm{~mA}$, (e) EL spectra of an SQS ${ }^{\mathrm{Ph}}$ mixture- and YAG:Ce phosphor-based white LED under various forward bias currents, (f) temporal luminous efficacy of various down-convertor- and YAG:Ce phosphor-based white LEDs operated at $60 \mathrm{~mA} 6$ months after fabrication.

ated instead of QDs along with the YAG:Ce phosphor, the Ra values fluctuated slightly down and up, whereas the LEs were slightly improved in both LEDs $\left(42.5 \mathrm{~lm} \mathrm{~W}^{-1}\right.$ for $\mathrm{SQ}_{1} \mathrm{~S}$ and 39.9 $\operatorname{lm} \mathrm{W}^{-1}$ for $\left.\mathrm{SQ}_{2} \mathrm{~S}\right)$. The incorporation of a mixture of $\mathrm{SQ}_{1} \mathrm{~S}$ and $\mathrm{SQ}_{2} \mathrm{~S}$ instead of QDs resulted in better optical properties $(\mathrm{Ra}=$ 80.0 and $\mathrm{LE}=44.1 \mathrm{~lm} \mathrm{~W}^{-1}$ ) than the cases incorporating a single product, $\mathrm{SQ}_{1} \mathrm{~S}$ or $\mathrm{SQ}_{2} \mathrm{~S}$. When $\mathrm{SQ}_{1} \mathrm{~S}^{\mathrm{Ph}}$ and $\mathrm{SQ}_{2} \mathrm{~S}^{\mathrm{Ph}}$ were incorporated instead of each QD, the LEs of the corresponding LEDs were distinctly improved to $50.5 \mathrm{~lm} \mathrm{~W}^{-1}$ and $47.8 \mathrm{~lm}$ $\mathrm{W}^{-1}$, respectively, as we reasoned by functional group matching and their $\mathrm{Ra}$ values fluctuated slightly down and up similar to the cases of SQSs. Most importantly, when the mixture of $\mathrm{SQ}_{1} \mathrm{~S}^{\mathrm{Ph}}$ and $\mathrm{SQ}_{2} \mathrm{~S}^{\mathrm{Ph}}$ was incorporated instead of QDs, the LE of the corresponding LED was markedly improved 
to $58.2 \mathrm{~lm} \mathrm{~W}^{-1}$ with a moderate increase of Ra to 81.8 . Therefore, replacing QDs with $\mathrm{SQS}^{\mathrm{Ph}} \mathrm{S}$ yielded a significant improvement in the LED performance, especially in the LE, and it was achieved through enhanced PL, better mixing with the resin, and better adhesion to the resin. This improvement was consistently observed throughout the repeated experiments.

The electroluminescence (EL) spectra showing the remarkable change are displayed in Fig. 2c. The EL spectrum of the white LED incorporating the mixture of $\mathrm{SQ}_{1} \mathrm{~S}^{\mathrm{Ph}}$ and $\mathrm{SQ}_{2} \mathrm{~S}^{\mathrm{Ph}}$ exhibits higher EL intensity than those of the other samples, whereas that of $\mathrm{SQ}_{2} \mathrm{~S}$ exhibits lower EL intensity. The lower EL intensity of the white LED incorporating $\mathrm{SQ}_{2} \mathrm{~S}$ is attributable to the aggregation of $\mathrm{SQ}_{2} \mathrm{~S}$ particles in the resin due to bad miscibility and adhesion. In particular, the eye-visible aggregated $\mathrm{SQ}_{2} \mathrm{~S}$ particles (indicated by arrows in Fig. 2a) can scatter the emitting light back and seriously lower the EL intensity at $\sim 550 \mathrm{~nm}$ as well as at $\sim 620 \mathrm{~nm}$ which correspond to the emission bands of YAG:Ce phosphors and $\mathrm{SQ}_{2} \mathrm{~S}$, respectively. In contrast, the higher EL intensity of the white LED incorporating the mixture of $\mathrm{SQ}_{1} \mathrm{~S}^{\mathrm{Ph}}$ and $\mathrm{SQ}_{2} \mathrm{~S}^{\mathrm{Ph}}$ is attributable to good miscibility and adhesion between $\mathrm{SQS}^{\mathrm{Ph}}$ and resins by functional group matching as shown in Fig. 2a. In particular, the EL intensity of the white LED incorporating the mixture of $\mathrm{SQ}_{1} \mathrm{~S}^{\mathrm{Ph}}$ and $\mathrm{SQ}_{2} \mathrm{~S}^{\mathrm{Ph}}$ is higher than that incorporating the single product $\mathrm{SQ}_{1} \mathrm{~S}^{\mathrm{Ph}}$ or $\mathrm{SQ}_{2} \mathrm{~S}^{\mathrm{Ph}}$. It occurred in the case of SQS too (Fig. S3†). Besides, when the relative ratio of the single product $\mathrm{SQ}_{2} \mathrm{~S}$ was increased from 0.7 to 1 , the EL intensity at $\sim 550 \mathrm{~nm}$ decreased but that at $\sim 620 \mathrm{~nm}$ increased as expected through the increased reabsorption of yellow light and emission of red light by the increased amount of $\mathrm{SQ}_{2} \mathrm{~S}$ (Fig. S3 ${ }^{\dagger}$ ), and the resultant LE has decreased from $39.9 \mathrm{~lm} \mathrm{~W}^{-1}$ to $30.8 \mathrm{~lm} \mathrm{~W}^{-1}$. A plausible reasoning for the higher EL in the case of the mixture compared to the case of a single product is that the concentration of $\mathrm{SQ}_{1} \mathrm{~S}$ (or $\mathrm{SQ}_{1} \mathrm{~S}^{\mathrm{Ph}}$ ) is a half of $\mathrm{SQ}_{2} \mathrm{~S}$ (or $\mathrm{SQ}_{2} \mathrm{~S}^{\mathrm{Ph}}$ ) in the current white LEDs and the reabsorption of $\sim 611 \mathrm{~nm}$ light by $\mathrm{SQ}_{2} \mathrm{~S}$ (or $\mathrm{SQ}_{2} \mathrm{~S}^{\mathrm{Ph}}$ ) may not be efficient in such a diluted medium. Thus, the resultant EL spectrum can be higher and broader than those incorporating a single product at the fixed concentration of total SQS analogues (100:10:0.70 or 0.75 for the weight ratio of resin: YAG:Ce :SQS analogues, 0.70 for the single product and 0.75 for the mixture). This reasoning was indirectly supported by a simplified test with SQS under excitation at $550 \mathrm{~nm}$ (Fig. S4 $\dagger$ ). The PL spectrum of the mixture solution of $\mathrm{SQ}_{1} \mathrm{~S}$ and $\mathrm{SQ}_{2} \mathrm{~S}\left[(2.5+5.0) \times 10^{8} \mathrm{M}\right]$ was higher and broader than that of $\mathrm{SQ}_{2} \mathrm{~S}$ only solution $\left(7.0 \times 10^{8} \mathrm{M}\right)$ and the integrated PL ratio was $1.4: 1$, which is far higher than the concentration ratio of $1.1: 1$. Thus, at the ratio 0.70 for the single SQS analogue, a concentration quenching may occur and the adjusted white light is emitted. On the other hand, at the combined ratio 0.75 for the mixture, the concentration quenching is minimized and the resultant white light is emitted. An image of the white light obtained from the LED with $\mathrm{SQS}^{\mathrm{Ph}}$ mixture under a forward bias current of $60 \mathrm{~mA}$ is included as an inset.

The Commission Internationale de l'Eclairage (CIE) colour coordinates $^{33}$ of LED VII $(0.3516,0.3702)$, VIII $(0.3431,0.3697)$, and IX $(0.3360,0.3355)$ incorporating $\mathrm{SQ}_{1} \mathrm{~S}^{\mathrm{Ph}}, \mathrm{SQ}_{2} \mathrm{~S}^{\mathrm{Ph}}$, and their mixture respectively are displayed in Fig. 2d. The white light of LED IX exhibits the highest Ra of 81.8 with a correlated colour temperature (CCT) of $5338 \mathrm{~K}$. As displayed in Fig. S5, $\dagger$ the respective values of $\mathrm{R} 1$ and $\mathrm{R} 8$, which are related to red colour rendering, were improved from 64.4 and 55.9 for YAG: Ce phosphor-based white LED to 76.8 and 69.4 by the addition of $\mathrm{QD}_{2}$, and further improved to 88.1 and 77.6 by the addition of the mixture of $\mathrm{SQ}_{1} \mathrm{~S}^{\mathrm{Ph}}$ and $\mathrm{SQ}_{2} \mathrm{~S}^{\mathrm{Ph}}$.

The PL bands of LED IX in Fig. 2e exhibit increasing intensities with increasing applied forward currents, implicating no saturation in their PL and thus a promising property for white LEDs. The operational EL stability of the white LED fabricated using each representative material was investigated 6 months after preparation. Notably, after 6 months, all the LED samples exhibited increased EL intensities compared with the corresponding as-prepared LED samples (Fig. S6†); the samples were then, slightly degraded for the initial several hours and then stabilized, though not perfectly as observed in the LEs and normalized EL intensities in Fig. $2 \mathrm{f}$ and S6. $\uparrow$ The initial optical degradation is attributable to the oxygen and moisture contained in the integration sphere, where they can permeate into the QDs through the porous silica. Nevertheless, the stabilization of QDs by silica under LED operation was remarkable, which is consistent with other reports. ${ }^{9-11}$ The order of operational EL stability was $\mathrm{SQS}^{\mathrm{Ph}}>\mathrm{SQS}>\mathrm{QD}$. After $60 \mathrm{~h}$ operation, compared with $I / I_{0}=0.91$ for $\mathrm{QD}_{2}$, the protection of QDS by SQS hybridization resulted in $I / I_{0}=0.95$, which was further improved to 0.98 for $\mathrm{SQS}^{\mathrm{Ph}}$. The hydrophobic stackable phenyl groups dangling from the $\mathrm{SQS}^{\mathrm{Ph}}$ appear to additionally protect the QDs from oxygen and moisture.

\section{Conclusions}

The newly designed QD-layer-encapsulated and phenyl-functionalized silica $\left(\mathrm{SQS}^{\mathrm{Ph}}\right.$ ) spheres exhibit remarkably enhanced PL and improved miscibility with and good adhesion to resin because of the gradual refractive index matching, with exclusion of FRET between QDs, and functional group matching through their characteristic hierarchical nanostructure and yield a white LED with significantly improved luminous efficiency and operational EL stability based on their original QDs. To the authors' knowledge, this is the first report that effectively introduces to QDs the gradual refractive index matching and functional group matching effect through a hierarchical nanostructure. Besides, the resultant SQS analogues can be a promising material for various applications including bio-imaging and bio-sensing due to their remarkably enhanced photoluminescence and stability and versatility in surface engineering.

\section{Acknowledgements}

This research was supported by the Future-based Technology Development Program (Green Nano Technology Development 
Program) and the Pioneer Research Center Program through the National Research Foundation of Korea funded by the Ministry of Science, ICT \& Future Planning (Grant No. 2014-060222 and NRF-2013M3C1A3065040) and the KIST institutional program (Project No. 2E25373).

\section{References}

1 O. Chen, J. Zhao, V. P. Chauhan, J. Cui, C. Wong, D. K. Harris, H. Wei, H.-S. Han, D. Fukumura, R. K. Jain and M. G. Bawendi, Nat. Mater., 2013, 12, 445.

2 Y. Shirasaki, G. J. Supran, M. G. Bawendi and V. Bulović, Nat. Photonics, 2013, 7, 13.

3 S. Jun and E. Jang, Angew. Chem., Int. Ed., 2013, 52, 679.

4 J. Lim, S. Jun, E. Jang, H. Baik, H. Kim and J. Cho, Adv. Mater., 2007, 19, 1927.

5 W. K. Bae, J. Kwak, J. W. Park, K. Char, C. Lee and S. Lee, Adv. Mater., 2009, 21, 1690.

6 E. Jang, S. Jun, H. Jang, J. Lim, B. Kim and Y. Kim, Adv. Mater., 2010, 22, 3076.

7 K.-H. Lee, J.-H. Lee, W.-S. Song, H. Ko, C. Lee, J.-H. Lee and H. Yang, ACS Nano, 2013, 7, 7295.

8 H. S. Jang, H. Yang, S. W. Kim, J. Y. Han, S.-G. Lee and D. Y. Jeon, Adv. Mater., 2008, 20, 2696.

9 S. Jun, J. Lee and E. Jang, ACS Nano, 2013, 7, 1472.

10 W.-S. Song, J.-H. Kim and H. Yang, Mater. Lett., 2013, 111, 104.

11 E.-P. Jang, W.-S. Song, K.-H. Lee and H. Yang, Nanotechnology, 2013, 24, 045607.

12 M. Cho, K. Lim and K. Woo, Chem. Commun., 2010, 46, 5584.

13 H. Han, J.-C. Pyun, H. Yoo, H. S. Seo, B. H. Jung, Y. S. Yoo, K. Woo and M.-J. Kang, Anal. Chem., 2014, 86, 10157.

14 S. Nakamura and G. Fasol, The Blue Laser Diode: GaN Based Light Emitters and Lasers, Springer, Berlin, 1996.

15 H. V. Demir, S. Nizamoglu, T. Erdem, E. Mutlugun, N. Gaponik and A. Eychmüller, Nano Today, 2011, 6, 632.

16 H.-S. Chen, C.-K. Hsu and H.-Y. Hong, IEEE Photonics Technol. Lett., 2006, 18, 193.
17 S. Kim, T. Kim, M. Kang, S. K. Kwak, T. W. Yoo, L. S. Park, I. Yang, S. Hwang, J. E. Lee, S. K. Kim and S.-W. Kim, J. Am. Chem. Soc., 2012, 134, 3804.

18 A. Aboulaich, M. Michalska, R. Schneider, A. Potdevin, J. Deschamps, R. Deloncle, G. Chadeyron and R. Mahiou, ACS Appl. Mater. Interfaces, 2014, 6, 252.

19 X. Wang, X. Yan, W. Li and K. Sun, Adv. Mater., 2012, 24, 2742.

20 D. L. Pavia, G. M. Lampman and G. S. Kriz Jr., Introduction to Spectroscopy: A Guide for Students of Organic Chemistry, W. B. Saunders, Philadelphia, 1979.

21 T. Fujii, Y. Gao, R. Sharma, E. L. Hu, S. P. DenBaars and S. Nakamura, Appl. Phys. Lett., 2004, 84, 855.

22 M. F. Schubert, D. J. Poxson, F. W. Mont, J. K. Kim and E. F. Schubert, Appl. Phys. Express, 2010, 3, 082502.

23 M. Jung, J. H. Kim, S. Lee, B. J. Jang, W. Y. Lee, Y.-M. Oh, S.-W. Park and D. Woo, J. Nanosci. Nanotechnol., 2012, 12, 5747.

24 R. Halir, P. J. Bock, P. Cheben, A. Ortega-Moñux, C. Alonso-Ramos, J. H. Schmid, J. Lapointe, D.-X. Xu, J. G. Wangüemert-Pérez, Í. Molina-Fernández and S. Janz, Laser Photonics Rev., 2015, 9, 25.

25 W. Stöber and A. Fink, J. Colloid Interface Sci., 1968, 26, 62.

26 L. L. Hench and J. K. West, Chem. Rev., 1990, 90, 33.

27 M. P. Lettinga, M. A. M. J. van Zandvoort, C. M. van Kats and A. P. Philipse, Langmuir, 2000, 16, 6156.

28 S. Dembski, C. Graf, T. Krüger, U. Gbureck, A. Ewald, A. Bock and E. Rühl, Small, 2008, 4, 1516.

29 W. W. Yu, L. Qu, W. Guo and X. Peng, Chem. Mater., 2003, 15, 2854.

30 W. Park, M. J. Kim, Y. Choe, S. K. Kim and K. Woo, J. Mater. Chem. B, 2014, 2, 1938.

31 B. N. Pal, Y. Ghosh, S. Brovelli, R. Laocharoensuk, V. I. Klimov, J. A. Hollingworth and H. Htoon, Nano Lett., 2012, $12,331$.

32 A. L. Rogach, D. Nagesha, J. W. Ostrander, M. Giersig and N. A. Kotov, Chem. Mater., 2000, 12, 2676.

33 W. J. Benjamin, Borish's Clinical Refraction, W. B. Saunders, Philadelphia, 1998. 